Figure 1. Mammary hibernoma, typical variant. Representative micrographs of the lesion composed of pale multivacuolated brown adipose cells with no atypia and mitotic figures (a, hematoxylin and eosin). The cells displayed S100 protein immunoexpression (b) and were negative for CD68 (c). Original magnification $\times 20$. [Color figure can be viewed at wileyonlinelibrary.com].

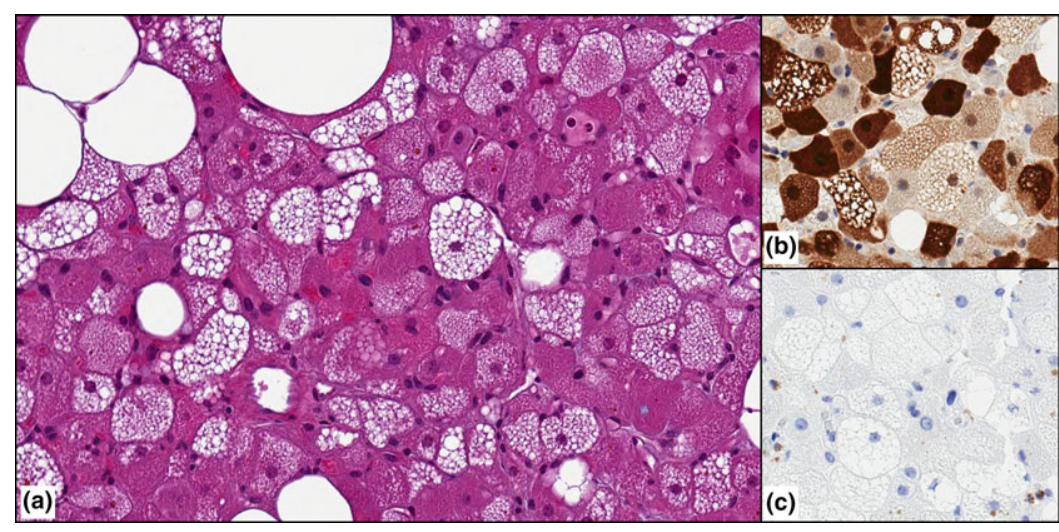

uncommonness, identification of mammary hibernomas can be challenging as they may resemble fat necrosis, xanthogranulomatous mastitis, granular cell tumor, lipoblastoma, and histiocytoid carcinoma.

Here, we illustrate a pure typical mammary hibernoma in a postmenopause woman with a soft mass in the upper-outer quadrant of the left breast. No other relevant clinical conditions were present. The lesion measured $4.3 \mathrm{~cm}$ in greatest dimension and was composed of an abnormal population of monomorphic multivacuolated S100-positive, CD68-negative cells with no atypia that resembled normal brown adipose tissue (Fig. 1). Surgical margins were clean and after 23 months of follow-up the patient is disease-free.

To date, only five pure mammary hibernomas have been reported in literature, therefore, their pathogenesis is poorly understood. Despite hibernoma can arise in any site where brown fat persists, such as posterior neck and upper back and shoulders, the most common primary site is represented by the thigh, an abnormal location for brown fat. For this reason, it has been hypothesized that mesenchymal precursors have the potential to differentiate into brown adipose cells in extra-brown fat locations, as in the breast. Largecohort molecular studies, coupled with follow-up data, are needed to start approaching the biology underpinning mammary hibernoma and to address the operational implications of these uncommon diagnosis for surgeons and pathologists.

\section{CONFLICT OF INTEREST}

The authors declare no competing financial interests.

\title{
Extranodal Rosai-Dorfman Disease of the Breast
}

\author{
Julie M. Jorns, MD
}

\author{
Department of Pathology, University of Michigan, Ann Arbor, Michigan
}

A middle-aged woman was noted to have scattered densities and asymmetry on routine mammogram. Diagnostic mammography and ultrasound

Address correspondence and reprint requests to: Julie M. Jorns, MD, Department of Pathology, University of Michigan, 1500 East Medical Center Drive 2G332 UH, Ann Arbor, MI 48109, USA, or e-mail: jjorns@med.umich. edu

DOI: $10.1111 /$ tbj.12690

(C) 2016 Wiley Periodicals, Inc., 1075-122X/16

The Breast Journal, Volume 23 Number 1, 2017 105-107 demonstrated a subcentimeter round mass with indistinct margins and no associated calcifications (Fig. 1). Computed tomography scan of the chest, abdomen, and pelvis demonstrated multiple subcutaneous nodules but no significant lymphadenopathy.

Breast core biopsy was performed and showed a multinodular lesion comprised of diffuse lymphoplasmacytic and histiocytic inflammation with both loose and well-formed granulomas, some of which were necrotizing. Similar findings were identified in a 

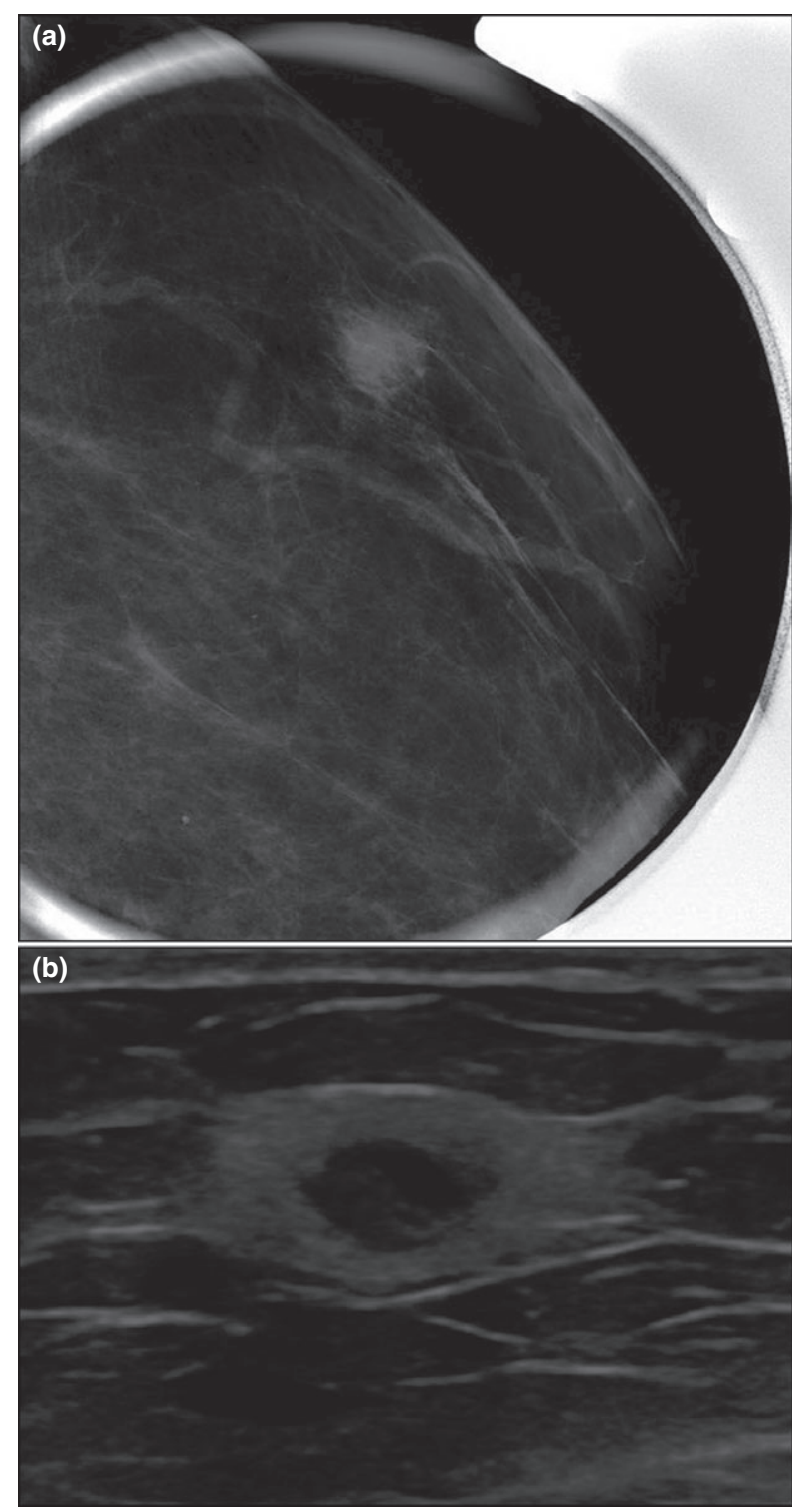

Figure 1. (a) Mammogram spot magnification and (b) ultrasound images of breast mass.

subcutaneous biopsy from the upper extremity. Immunohistochemical stains showed the histiocytes to express CD68, CD163, and S100 via immunohistochemical staining. Inflammatory cells within the cytoplasm of large histiocytes, or emperipolesis, were also highlighted by these stains (Fig. 2). Special stains for fungal and acid-fast organisms were negative. These features were diagnostic of extranodal Rosai-Dorfman disease (RDD).

RDD, also known as sinus histiocytosis with massive lymphadenopathy, is a benign histiocytic proliferation that predominantly involves lymph nodes, most often those of the head and neck regions. However, extranodal disease commonly accompanies lymph node involvement and has been identified throughout the body, with skin and soft tissue being the most frequent extranodal sites. Extranodal disease without identifiable lymph node involvement has been estimated to occur in $23 \%$ of cases.

Extranodal RDD is a rare diagnosis in the breast. When it does involve the breast, it may be either unilateral or bilateral. Extranodal RDD involving the breast has variable imaging findings but may mimic breast cancer clinically and radiographically, presenting as a mass as in this case.

Diagnostic features on biopsy are characteristic histiocytes with abundant pale cytoplasm exhibiting emperipolesis and co-expression of $\mathrm{S} 100$ and macrophage markers such as CD68 and CD163. There is often abundant associated lymphoplasmacytic inflammation. While the latter features were prominent in this case, there was also an unusual finding of necrotizing granulomas, which necessitated additional evaluation for fungal and acid-fast microorganisms.

RDD has been linked with infectious agents such as Epstein-Barr virus, human herpesvirus, and parvovirus B19, as well as with IgG4-related diseases and with germline mutations in SLC29A3 in familial cases. RDD may show spontaneous regression in $20 \%$ of cases and is typically only treated when there is need for symptomatic relief, with therapies including steroids, immunomodulatory and chemotherapeutic drugs, radiotherapy, and surgical excision. At the time of this report, this patient had no significant progression of disease and management was observation only.

\section{Acknowledgment}

Radiographic images are courtesy of Dr. Leah Carlson, Department of Radiology, University of Michigan. 

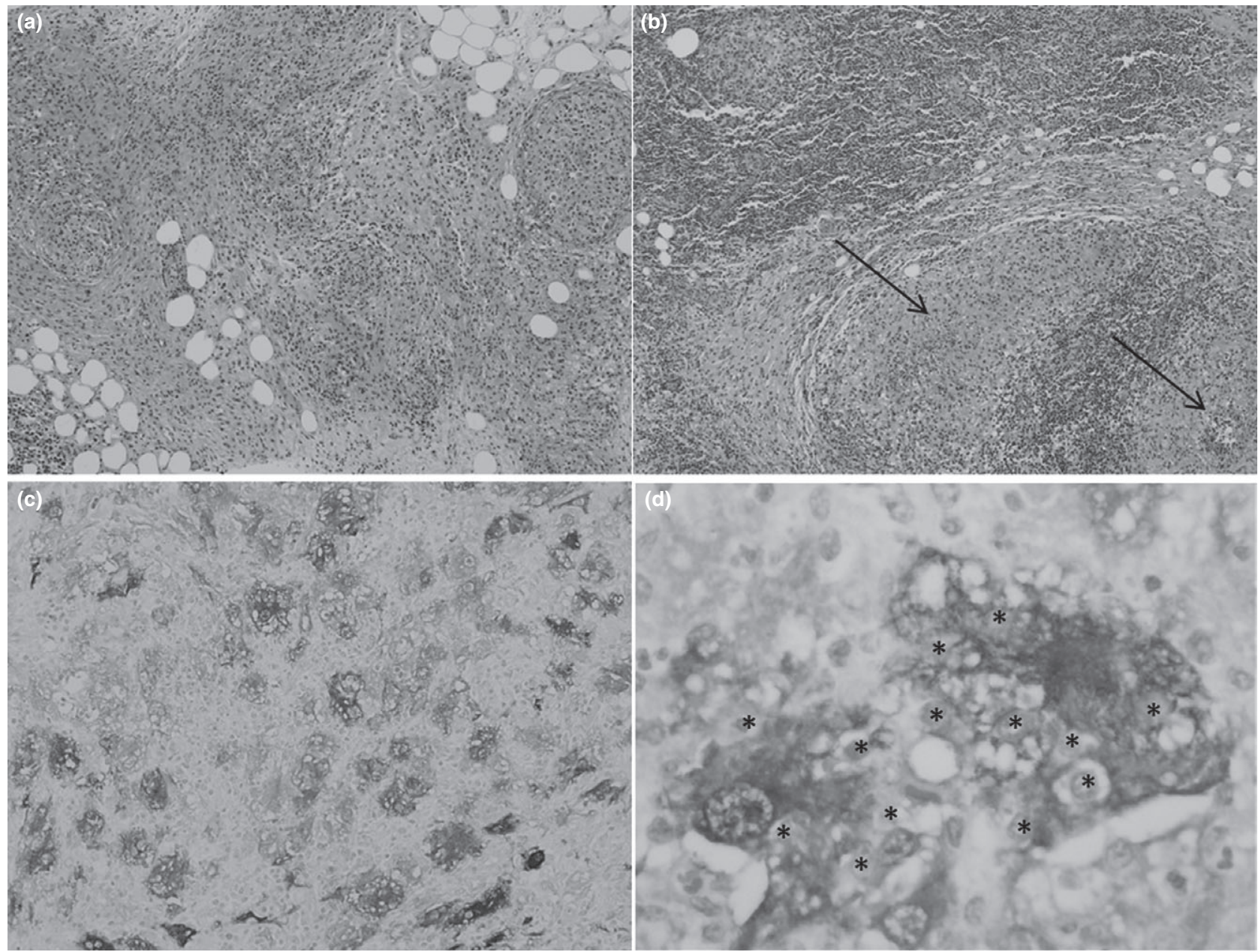

Figure 2. ( $a$ and $b)$ Breast biopsy showing lymphoplasmacytic and histiocytic inflammation with granulomas, including necrotizing granulomas (arrows, b) $(\mathrm{H \& E}, 10 \times)$. (c) S100-positive histiocytes (IHC, 20x). (d) S100-positive histiocytes with prominent emperipolesis (inflammatory cells within histiocyte cytoplasm are denoted by *s) (IHC, 60x).

\section{Molluscum Contagiosum: A Rare Nipple Lesion}

\section{Charlotte Ives, MBBS, MD, $\stackrel{*}{ }$ Michael Green, MBChB, $\stackrel{*}{*}$ and Tanwen Wright, $\mathrm{MBBS}^{\dagger}$}

*Breast Unit, South Devon Healthcare NHS Foundation Trust, Torquay, Devon, UK; †Histopathology Unit, South Devon Healthcare NHS Foundation Trust, Torquay, Devon, UK

Address correspondence and reprint requests to: Charlotte Ives, Breast Unit, South Devon Healthcare NHS Foundation Trust, Torquay, Devon TQ2 7AA, UK, or e-mail: charlotte.ives@nhs.net

DOI: $10.1111 /$ tbi.12693

(C) 2016 Wiley Periodicals, Inc., 1075-122X/16

The Breast Journal, Volume 23 Number 1, 2017 107-108
$\mathrm{M}$ olluscum contagiosum (MC) is a common selflimiting viral illness caused by a poxvirus. It classically affects children aged one to five with small flesh-colored skin lesions that have a central umbilication. They usually appear in clusters and can migrate 